\title{
Habilidades lingüísticas decodificación en niños con Trastorno Específico del Lenguaje con y sin dificultades de comprensión lectora
}

\author{
Linguistic skills and decoding among children with \\ Specific Language Impairment with and without \\ reading comprehension difficulties
}

María Antonia Reyes

Departamento de Fonoaudiología Universidad de Chile

\section{Zulema De Barbieri}

Departamento de Fonoaudiología Universidad de Chile
Contacto con el autor: María Antonia Reyes Santiago - Chile Correo-e: mariareyes@med.uchile.cl

Recibido: 05/04/2018 Aceptado: 30/08/2018

\begin{abstract}
RESUMEN
Los niños con TEL son considerados un grupo de riesgo para el aprendizaje del lenguaje escrito, dado que sus dificultades lingüísticas pueden influir en su desempeño en comprensión lectora, aunque esto no necesariamente ocurre en todos los niños con TEL. El objetivo de este estudio es comparar el desempeño en las habilidades lingüísticas y decodificación entre niños con TEL que se agruparon según la presencia o ausencia de dificultades en comprensión lectora. La muestra estuvo constituida por 60 niños con TEL de primer año básico, 42 de ellos con problemas de comprensión lectora y 18 niños que no presentaban dificultad en este aspecto. Se evaluaron habilidades lectoras (decodificación y comprensión lectora) y habilidades lingüísticas (conciencia fonológica, vocabulario y discurso narrativo expresivo y comprensivo). Los resultados muestran que no existen diferencias estadísticamente significativas en las habilidades lingüísticas entre niños con y sin dificultades de comprensión lectora. En cuanto a la decodificación, se observa un rendimiento significativamente inferior en el grupo de niños con dificultades de comprensión lectora. En este grupo, conciencia fonológica y decodificación están altamente correlacionadas con la comprensión lectora, mientras que estas mismas variables no se correlacionan en los niños sin problemas de comprensión lectora.
\end{abstract}

Palabras clave: Trastorno específico del lenguaje, habilidades lingüísticas, decodificación, comprensión lectora.

\begin{abstract}
Children with Specific Language Impairment (SLI) are deemed to be a group at risk in learning written language. Their linguistic deficit may impact on their reading comprehension performance, although this not the case for all children with SLI. The aim of this study is to observe the possible differences in linguistic and decoding skills in children with SLI with and without reading comprehension problems. The sample consisted of 60 first grade children with SLI, of which 42 are poor comprehenders and 18 are good comprehenders. Reading skills (decoding and reading comprehension) and linguistic skills (phonological awareness, vocabulary as well as expressive and comprehensive narrative speech) were assessed. Results show that there are no statistically significant differences in linguistic skills between good comprehenders and poor comprehender. Significant differences were found for decoding, with children with reading comprehension problems performing significantly lower than good comprehenders. In the group of children with reading comprehension problems, phonological awareness and decoding highly correlate with reading comprehension, while the very same variables have no correlation among children with good reading comprehension.
\end{abstract}

Keywords: Specific language impairment, linguistic skills, decoding, reading comprehension. 


\section{Introducción}

El Trastorno Específico del Lenguaje (TEL) es uno de los cuadros más frecuentes de problemas en la comunicación oral en la población infantil. Los niños con este tipo de dificultades no evidencian pérdida auditiva, daño cerebral, desempeños cognitivos descendidos ni déficit motor (Acosta, 2012). Se caracterizan por ser un grupo heterogéneo en virtud de las diversas dificultades lingüísticas que pueden presentar en los distintos niveles del lenguaje, ya sea a nivel expresivo o comprensivo y expresivo (Marinis, 2011; Sanz-Torrent, Andreu, Badia, \& Serra, 2010). Asimismo, se ha evidenciado que sus dificultades lingüísticas pueden perdurar hasta la educación básica e incluso persistir en la edad adulta (Guasti, 2002).

Los niños con TEL son considerados un grupo de riesgo para el aprendizaje del lenguaje escrito, debido a que sus dificultades lingüísticas $y$ metalingüísticas pueden repercutir en su desempeño lector (Bishop \& Adams, 1990; Ricketts, 2011; Snowling, Bishop, \& Stothard, 2000). Estos niños presentarían un menor desempeño en ciertas habilidades necesarias para el desempeño lector, como son la conciencia fonológica, el léxico y el discurso narrativo (Coloma \& De Barbieri, 2007; Dickinson, McCabe, Anastasopoulos, PeisnerFeinberg, \& Poe, 2003; Nation \& Snowling, 2004).

Una posible explicación a las dificultades lectoras de los niños con TEL proviene de la teoría de la Visión Simple de la Lectura (VSL). Esta teoría plantea que la comprensión lectora es el resultado de la decodificación y de la comprensión oral (Protopapas, Simos, Sideridis, \& Mouzaki, 2012). Si la decodificación y la comprensión oral contribuyen de manera independiente a la comprensión lectora, cada una debiese estar basada en diferentes habilidades subyacentes. La conciencia fonológica provee la base para la decodificación mientras que los aspectos lingüísticos como el vocabulario, la gramática y el discurso son relevantes para la comprensión oral (Balbi, Cuadro, \& Trías, 2009; Hoover \& Gough, 1990; Oakhill \& Cain, 2012).

Por otra parte, no todos los niños con TEL que presentan problemas en comprensión lectora manifiestan dificultades en la decodificación (Balbi et al., 2009; Catts, Fey, Tomblin, \& Zhang, 2002). En un estudio de Conti-Ramsden, Botting, Simkin, \& Knox (2001) se evidenció que el $77 \%$ de niños con TEL de 11 años presentaba dificultades en reconocimiento de palabras aisladas mientras que el 98\% presentaban problemas de comprensión lectora. Por tanto, parece ser que existe un mayor porcentaje de niños con problemas en comprensión lectora que en el reconocimiento de palabras aisladas.

$\mathrm{Si}$ bien es frecuente que los niños con TEL presenten dificultades en comprensión lectora, no se da en todos los casos. Catts et al. (2002) evaluaron, en un estudio longitudinal, habilidades lectoras en escolares con TEL en segundo y cuarto básico. En segundo básico el $48,1 \%$ de ellos evidenciaron dificultades de comprensión lectora y en cuarto básico un 35,9\% mantuvo estos problemas. Mesa, Tirado, \& Saldaña (2013) mostraron que el 75\% de niños con TEL de habla española entre 7 y 12 años presentaba dificultades de comprensión lectora. Coloma et al. (2012) encontraron que el $67 \%$ de escolares de primero básico con TEL tenía 
dificultades en comprensión lectora, mientras que un $33 \%$ presentó rendimientos apropiados en esta habilidad.

Los estudios mencionados dan cuenta de que un número importante de niños con TEL presentan dificultades en la comprensión lectora, pero también de que existe un grupo con un buen desempeño en este aprendizaje.

El presente estudio tiene por objetivo comparar niños diagnosticados con TEL con y sin dificultades en comprensión lectora. La comparación se realizará sobre la base de sus respectivos desempeños en habilidades lingüísticas y de decodificación.

Estudiar este aspecto en los niños con TEL cobra relevancia ya que los niños ingresados por diagnóstico de TEL Mixto o Expresivo a los programas de integración escolar (PIE) en Chile representan el $18 \%$, constituyendo el tercer diagnóstico más frecuente de todos los niños integrados (Fundación Chile \& Centro de Estudios MINEDUC, 2013).

\section{Método}

La presente investigación corresponde a un estudio no experimental, cuyo diseño es transversal de tipo descriptivo, comparativo y correlacional.

\section{Participantes}

En esta investigación participaron $60^{1}$ escolares con diagnóstico de TEL Mixto de primer año básico, pertenecientes a escuelas regulares con proyectos de integración de establecimientos municipales y

\footnotetext{
${ }^{1}$ Los participantes y sus evaluaciones pertenecen a un grupo de una muestra previa obtenida en el marco del proyecto FONDECYT N¹130201.
}

particulares subvencionados de la Región Metropolitana de Santiago de Chile. Todos presentaban habilidades cognitivas no verbales $\mathrm{y}$ audición dentro de rangos normales.

Los escolares se distribuyeron en dos grupos, uno conformado por 42 niños con TEL que presentaban dificultades en la comprensión lectora ( 25 niños y 17 niñas, con promedio de edad de 6 años 10 meses) y otro conformado por 18 niños con TEL que no presentan dificultades en comprensión lectora (8 niños y 10 niñas, con un promedio de edad de 6 años 5 meses).

Para conformar ambos grupos y distinguir entre niños con TEL con y sin dificultades de comprensión lectora, se utilizó la prueba Comprensión de textos de la batería "Language Survey -Revised" (versión en español) de Woodcock, Muñoz, Ruef, \& Alvarado (2005). Esta prueba define un buen lector cuando se ubica en los niveles de lectura manejable o fácil. Por su parte, el mal lector es aquel que se ubica en el nivel de dificultad lectora. Los niveles se determinan a partir del puntaje obtenido en la prueba.

\section{Selección de los participantes}

Los participantes de este estudio se seleccionaron a partir del diagnóstico que realizó el profesional fonoaudiólogo del establecimiento escolar. Dicho diagnóstico se basa en criterios estándares propuestos por el Ministerio de Educación de Chile, y requiere que el niño presente rendimientos deficitarios en comprensión y/o en expresión del lenguaje en las pruebas propuestas por el Decreto Supremo 170/2010 (Ministerio de Educación, 2010). A partir de esta información, se 
eligieron los escolares con TEL que presentaban dificultades del lenguaje tanto a nivel expresivo como comprensivo. Cabe señalar que las 25 escuelas participantes se seleccionaron mediante un tipo de muestreo aleatorio simple entre las escuelas municipales y particulares subvencionadas de la Región Metropolitana.

Con el fin de descartar dificultades cognitivas y auditivas, se aplicó el Test de Matrices Progresivas, Escala Coloreada de (Raven, 2005). El desempeño auditivo se midió mediante una audiometría de barrido en las frecuencias 500, 1000, 2000 y $4000 \mathrm{~Hz}$. Se utilizaron los criterios internacionales propuestos por la American Speech Language Hearing Association (2005).

La evaluación de las habilidades lingüísticas y lectoras se efectuó individualmente en una sala tranquila en el establecimiento educacional. En el primer semestre se evaluaron las habilidades lingüísticas y en el segundo, las lectoras. Se realizaron dos sesiones para la evaluación de las habilidades lingüísticas y otras dos sesiones para la evaluación de las habilidades lectoras (decodificación y comprensión lectora).

Todos los participantes fueron autorizados por sus padres mediante la firma de un consentimiento informado. Además, cuenta con la aprobación del Comité de Ética de la Investigación de la Facultad de Medicina de la Universidad de Chile Nº45-2012.

\section{Instrumentos}

En primer término, se presenta el instrumento utilizado para conformar ambos grupos. Luego, se exponen los instrumentos utilizados para la evaluación de la decodificación y las habilidades lingüísticas.

\section{Prueba para conformar los grupos}

\section{Comprensión lectora}

Esta habilidad se midió a través de la prueba "Comprensión de textos", de la batería Language Survey - Revised (Woodcock et al., 2005). Esta prueba mide compresión de palabras, oraciones y textos. Comienza con ítems que evalúan la habilidad de relacionar la palabra con un dibujo del objeto que se representa. En los siguientes ítems se asocia un dibujo a una frase. Posteriormente, se presentan oraciones con distinto grado de complejidad sintáctica que requieren ser completadas con una palabra. Luego, aparecen textos que progresivamente se hacen más difíciles en su sintaxis y en su léxico, los que también deben ser completados con una palabra que les otorgue sentido. Es importante destacar que para realizar las tareas de esta prueba se requiere un buen manejo de la decodificación, ya que no existe ningún tipo de clave que apoye la comprensión.

A las respuestas correctas se les asigna 1 punto y la máxima puntuación que se puede alcanzar es 32 puntos. La confiabilidad es de .95 , según la prueba de Kuder y Richardson.

Instrumentos utilizados para la evaluación de la decodificación y de las habilidades lingüísticas

\section{Decodificación}

Esta habilidad fue evaluada mediante la prueba de "Identificación de letras y palabras" de la batería 
Language Survey - Revised (Woodcock et al., 2005). La prueba está constituida por 75 ítems, de los cuales los primeros corresponden a la identificación de letras, mientras que los siguientes refieren a palabras que se deben leer fluidamente. La complejidad de los ítems está determinada por su grado de familiaridad. Es decir, las palabras son progresivamente menos frecuentes a medida que el test avanza. En esta prueba, el niño debe identificar letras en distintos contextos: entre dibujos, entre otras letras o entre números. Luego, debe reconocer palabras, leer términos progresivamente más complejos en los planos de la fonología, la ortografía y el significado. A las respuestas correctas se les asigna 1 punto, con una puntuación máxima de 75 puntos. Su confiabilidad es de .96 , de acuerdo a la prueba de Kuder y Richardson.

Finalmente, es necesario mencionar que las pruebas de decodificación y comprensión lectora son parte de una misma batería, por lo que poseen criterios similares para su utilización. Se aplican por páginas completas y se suspenden cuando el menor presenta un número de errores consecutivos según las indicaciones de cada prueba.

\section{Habilidades Lingüísticas}

En este punto se consideró: conciencia fonológica, discurso narrativo (comprensión y producción) y léxico. A continuación, se explican las pruebas utilizadas para la evaluación de cada habilidad:

Conciencia Fonológica: esta habilidad metalingüística se evaluó mediante la Prueba de Conciencia Fonológica para $1^{\circ}$ básico de Himmel \& Infante (2007), la cual consta de 8 subpruebas que contienen 6 ítems cada una. Las dos primeras son actividades que requieren comparar fonemas, tanto iniciales como finales; las subpruebas tercera $y$ cuarta implican aislar fonemas iniciales y finales; la quinta supone realizar síntesis fonémica; la sexta y la séptima exigen omitir el fonema inicial o final. Por último, la octava subprueba demanda segmentar fonemas de la palabra escuchada. Su confiabilidad es .96 y se obtuvo mediante el coeficiente $\alpha$ Cronbach.

Discurso Narrativo: se estudió la comprensión y producción narrativa con el instrumento Evaluación del Discurso Narrativo (EDNA) de Pavez, Coloma, \& Maggiolo (2008) que contiene dos subpruebas para cada modalidad.

Para la producción narrativa, la prueba EDNA implica el recontado de tres cuentos de distinta complejidad estructural. Los relatos son: La ardillita glotona, El sapito saltarín y El lobo friolento. Los cuentos infantiles se analizan identificando tres categorías estructurales básicas (presentación, episodio y final) a las que se les asigna puntaje. Es necesario destacar que esta prueba no tiene un puntaje máximo, ya que la puntuación asignada depende del desempeño narrativo logrado por el niño. La prueba de producción presenta una confiabilidad, según el coeficiente $\alpha$ de Cronbach, de 77 .

Por otro lado, la prueba de comprensión de EDNA incluye tres cuestionarios, uno para cada cuento, con preguntas literales e inferenciales (La ardillita glotona: 6 preguntas literales y 3 inferenciales, El sapito saltarín y El lobo friolento: 7 preguntas literales y 3 inferenciales). Tanto las interrogantes literales como las inferenciales se relacionan con las categorías estructurales. Tiene una confiabilidad de 
.84 , según el coeficiente $\alpha$ de Cronbach.

Léxico: Se utilizó el instrumento "Vocabulario sobre dibujos", que corresponde a la batería Language Survey - Revised (Woodcock et al., 2005). Está compuesto por 58 ítems que aumentan progresivamente en dificultad, en la medida en que las palabras evaluadas son menos familiares para los niños. Cada ítem está ilustrado por una imagen que representa el término evaluado. Pese a que la prueba mide vocabulario en las modalidades de comprensión y producción, cabe destacar que 52 ítems corresponden al léxico expresivo. La prueba para evaluar léxico presenta una confiabilidad de .89, de acuerdo a la prueba de Kuder y Richardson.

\section{Análisis de los datos}

A nivel estadístico inferencial, se realizaron comparaciones entre grupos y pruebas de correlación. Primero, se efectuó la comparación del desempeño entre el grupo de niños con dificultades de comprensión lectora y el grupo de niños sin dificultades de comprensión lectora, considerando conciencia fonológica, léxico, comprensión y producción de discurso narrativo y decodificación. Para la comparación de los grupos, se seleccionaron aleatoriamente 18 de los 42 sujetos del grupo de niños con dificultades en comprensión lectora para equiparar la cantidad de participantes con el grupo de niños sin dificultades. Lo anterior para poder comparar ambos grupos mediante la aplicación de la prueba t de Student. Luego, se realizó el análisis de correlación para cada grupo entre la comprensión lectora y el resto de las habilidades evaluadas (conciencia fonológica, léxico, comprensión y producción de discurso narrativo y decodificación). Para ello, se consideró a la totalidad de los niños en cada grupo, es decir 42 y 18 participantes. Todos los análisis se realizaron mediante el paquete estadístico R (R Core Team, 2018).

A nivel descriptivo, se realizó una caracterización del nivel de comprensión lectora alcanzado por cada grupo.

\section{Resultados}

En primer término, se presentan los resultados del análisis cuantitativo de comparación de habilidades lingüísticas y de decodificación en niños con TEL, con y sin dificultades en comprensión lectora. Por último, se expone el análisis cualitativo del desempeño en compresión lectora de cada grupo.

\section{Análisis cuantitativo del desempeño de niños con TEL, con y sin dificultades en comprensión lectora}

La comparación se realizó mediante la prueba $\mathrm{t}$ de Student, considerando las medias de desempeño de ambos grupos de niños en las habilidades evaluadas. Los resultados obtenidos se aprecian en la Tabla 1, en la cual se muestran los estadísticos descriptivos media (M) y desviación estándar (D.S.) de las diferentes variables para cada grupo así como los valores de t y la significancia estadística. 
Tabla 1

Comparación de los desempeños en habilidades lingüísticas y decodificación entre niños con TEL con y sin dificultades de comprensión lectora.

\begin{tabular}{|c|c|c|c|c|c|c|}
\hline \multirow[b]{2}{*}{ Variables } & \multicolumn{2}{|c|}{$\begin{array}{l}\text { Niños con TEL con dificultades } \\
\text { de Comprensión Lectora } \\
\text { (n 18) }\end{array}$} & \multicolumn{2}{|c|}{$\begin{array}{l}\text { Niños con TEL sin dificultades de } \\
\text { Comprensión Lectora } \\
\text { (n 18) }\end{array}$} & \multirow[b]{2}{*}{$t$} & \multirow[b]{2}{*}{$p$} \\
\hline & $M$ & $D S$ & $M$ & $D S$ & & \\
\hline Conciencia fonológica & 8.19 & 4.97 & 10.89 & 8.3 & 1.28 & 0.1 \\
\hline Comprensión narrativa & 22.36 & 6.25 & 23.44 & 8.3 & 0.49 & 0.3 \\
\hline Producción narrativa & 6.89 & 4.49 & 8.78 & 5.35 & 1.3 & 0.1 \\
\hline Léxico & 28.74 & 4.66 & 29.89 & 5.68 & 0.7 & 0.2 \\
\hline Decodificación & 24.14 & 14.18 & 54.89 & 11 & 9 & $<0.001 * * *$ \\
\hline
\end{tabular}

Diferencia es significativa si $p<0.05 * * * p<.001$

Como se advierte en la Tabla 1, los niños con TEL y dificultades en la comprensión lectora presentan un desempeño significativamente inferior en decodificación que los escolares con TEL sin problemas en comprensión lectora $(\mathrm{t}=9 ; p<.001)$. No ocurre lo mismo al comparar las habilidades lingüísticas, ya que no existen diferencias estadísticamente significativas entre ambos grupos de niños con TEL.

Posteriormente, se indagó la posible relación entre la comprensión lectora y las habilidades lingüísticas y de decodificación, para cada grupo. Se consideraron los puntajes obtenidos en cada variable y se aplicó la prueba de correlación de Pearson. Los resultados obtenidos se aprecian en la Tabla 2.

Tabla 2

Correlaciones entre comprensión lectora y conciencia fonológica, léxico, comprensión narrativa, producción narrativa y decodificación en niños con TEL sin y con dificultades de comprensión lectora.

\begin{tabular}{lcc}
\hline & \multicolumn{2}{c}{ Comprensión lectora } \\
\cline { 2 - 3 } & $\begin{array}{c}\text { Niños con TEL sin dificultades de } \\
\text { comprensión lectora } \\
n: 18\end{array}$ & $\begin{array}{c}\text { Niños con TEL con dificultades de } \\
\text { comprensión lectora } \\
n: 42\end{array}$ \\
\hline Conciencia Fonológica & $r=.07$ & $r=.4^{* *}$ \\
Léxico & $r=.35$ & $r=-.06$ \\
Comprensión narrativa & $r=.25$ & $r=-.04$ \\
Producción narrativa & $r=.21$ & $r=.85^{* * *}$ \\
Decodificación & $r=.17$ & $r$
\end{tabular}

$* * p<.01 * * * p<.001$ 
Como se advierte en la Tabla 2, en el grupo de niños con TEL y dificultades en la comprensión lectora, se correlaciona esta habilidad con conciencia fonológica y decodificación $(r=.4 ; p<.01$ y $r=.85$; $p<.001$, respectivamente), resultando ambas correlaciones estadísticamente significativas. Estas correlaciones no están presentes en el grupo de niños con TEL sin dificultad en comprensión lectora. Sin embargo, en dicho grupo existe una relación ( $r=.35)$ entre léxico y comprensión lectora, aunque sin significancia estadística.

\section{Análisis cualitativo del perfil lector de los niños con TEL}

Al observar el rendimiento en comprensión lectora y de acuerdo con los ítems que logró responder cada niño en la prueba de comprensión de textos, se aprecia que 31 participantes (74\%) del grupo de niños con TEL considerados con dificultades en comprensión lectora lograron un desempeño de comprensión lectora solo a nivel de palabras, ya sea asociando la imagen con su concepto correspondiente o leyendo y completando frases, pero con apoyo de la imagen.

En el grupo de niños con TEL sin dificultades en comprensión lectora, 11 escolares (61\%), lograron un desempeño de comprensión lectora a nivel de enunciados simples sin apoyo visual y 7 (39\%) comprendieron oraciones complejas, lo cual es acorde a lo estipulado en la prueba y esperable para la edad. Los datos comentados se muestran en la Tabla 3.

Tabla 3

Distribución del rendimiento de cada grupo, en comprensión lectora de acuerdo con los ítems de la prueba.

\begin{tabular}{lccc}
\hline Nivel de comprensión & Ítem de la prueba & $\begin{array}{c}\text { Niños con TEL sin } \\
\text { dificultades en } \\
\text { comprensión lectora }\end{array}$ & $\begin{array}{c}\text { Niños con TEL con } \\
\text { dificultades en } \\
\text { comprensión lectora }\end{array}$ \\
\hline Asociación imagen concepto & $1-3$ & 16 \\
Completación de frases con apoyo de imagen & $4-6$ & 11 & 15 \\
Completación de oraciones simples & $7-12$ & 7 & 10 \\
Completación de oraciones más complejas & $13-20$ & 1 \\
\hline
\end{tabular}

\section{Discusión}

La presente investigación tuvo como objetivo comparar el desempeño en habilidades lingüísticas (conciencia fonológica, léxico, discurso narrativo) y de decodificación en niños con TEL con y sin dificultades de comprensión lectora.
En primer término, es importante señalar que de una muestra aleatoria de 60 niños diagnosticados con TEL, un gran porcentaje de ellos tuvo problemas de comprensión lectora $(70 \%$ del total de la muestra), lo cual evidencia que la mayoría de los niños con TEL presenta dificultades a este aspecto lector. Por consiguiente, se corrobora que 
constituyen un grupo de riesgo para el aprendizaje de la lectura, en particular para la comprensión lectora, tal como señalan diversas investigaciones (Bishop \& Adams, 1990; Mesa et al., 2013; Ricketts, 2011; Snowling et al., 2000).

Los resultados de este estudio, también señalan que existe un grupo de niños con TEL que, a pesar de presentar dificultades en las habilidades lingüísticas, no evidencian bajo desempeño en la comprensión lectora. La evidencia previa es coincidente con resultados de diversas investigaciones (Catts et al., 2002; Coloma et al., 2012; Coloma, Silva, Palma, \& Holtheuer, 2015; Coloma, Sotomayor, De Barbieri, \& Silva, 2015).

En relación a las diferencias que pudiesen existir entre niños con y sin dificultades de comprensión lectora, los hallazgos de esta investigación dan cuenta que no existen diferencias significativas entre los desempeños en habilidades lingüísticas entre ambos grupos. Este resultado es esperable, dado que todos los niños poseen diagnóstico de TEL como base, lo que implica desempeños disminuidos en algunos de los componentes lingüísticos evaluados. Lo anterior coincide con hallazgos de un estudio longitudinal, donde siguieron niños con TE entre kínder y tercero básico. En este trabajo no se encontraron diferencias significativas en ninguna tarea de lenguaje oral entre participantes con TEL con $y$ sin problemas en habilidades lectora (Vandewalle, Boets, Boons, Ghesquière, \& Zink, 2012). Esto sugiere que el déficit lingüístico en las etapas iniciales del aprendizaje lector no parece ser una variable que genere diferencias entre niños con TEL con y sin problemas de comprensión lectora.

A su vez, los resultados evidencian que la única habilidad que diferencia a ambos grupos de manera significativa es la decodificación, lo que es coherente con el estudio realizado en escolares con diagnóstico de TEL donde la comprensión lectora y la decodificación se relacionaron de forma significativa (Coloma, Sotomayor, et al., 2015). Ello insinúa que las dificultades de comprensión de lectura experimentadas por niños con TEL en etapas iniciales, son generalmente el resultado de procesos ineficientes de decodificación (Kelso, Fletcher, \& Lee, 2007).

En el grupo de niños con TEL y dificultades en comprensión lectora la conciencia fonológica y la decodificación se relacionan de manera significativa con la comprensión lectora, relación que no se evidencia en el grupo sin dificultades. La relación encontrada entre conciencia fonológica, decodificación y comprensión lectora es consistente con lo propuesto por el modelo de la Visión Simple de la Lectura (VSL), donde se plantea que las habilidades fonológicas constituyen la base de la decodificación (Hoover \& Gough, 1990; Oakhill \& Cain, 2012). Por consiguiente, en etapas iniciales del aprendizaje lector la decodificación jugaría un rol central en la comprensión lectora (Catts \& Kamhi, 2005; Ricketts, 2011).

Cabe señalar que el desempeño lector de los niños con TEL con dificultades en comprensión lectora sólo alcanzó niveles básicos en esta habilidad, correspondiente a los primeros ítems de la prueba utilizada para evaluar la comprensión lectora.

En los niños con TEL sin dificultades en comprensión lectora se observó una mayor relación entre léxico y comprensión lectora, aunque sin significación estadística. Es importante mencionar 
que este resultado se encuentra limitado por el tamaño de la muestra, por lo que un mayor número de participantes de niños con TEL sin dificultades de comprensión lectora podría entregar un resultado más confiable. A su vez, en este grupo de niños, hubo desempeños en comprensión lectora acorde a su edad, ya sea a nivel de enunciados simples sin apoyo visual y de oraciones complejas.

En síntesis, los resultados de este estudio sugieren que la conciencia fonológica y la decodificación pueden estar cumpliendo un rol importante en la comprensión lectora de los niños con dificultades, es decir que un mejor rendimiento especialmente en decodificación podría facilitar un mejor desempeño en la comprensión lectora en niños con TEL que cursan primer año básico.

El instrumento utilizado para evaluar comprensión lectora en la investigación puede ser interpretado como una limitación, ya que la prueba requiere un adecuado manejo de la decodificación. Contar con un instrumento que no dependa exclusivamente de la decodificación ayudaría a estudiar con mayor detalle las relaciones entre las habilidades lingüísticas y la comprensión lectora.

Finalmente, los resultados obtenidos en este estudio contribuyen a un mejor conocimiento de las dificultades lectoras que pueden evidenciar los niños con TEL. Lo anterior cobra relevancia porque permite ayudar a diseñar estrategias más específicas de estimulación de la comprensión lectora en escolares con este diagnóstico. Esto porque abordar aspectos como la conciencia fonológica y la decodificación en los planes de intervención, podría facilitar el proceso lector en etapas iniciales del aprendizaje.
Agradecimientos: se agradece el financiamiento otorgado por CONICYT a través de la Beca Nacional de Magíster año 2013, al FONDECYT Regular 2013 no1130201 y al Proyecto Basal FB0003 del Programa de Investigación Asociativa de CONICYT, Ministerio Educación, Chile.

\section{Referencias}

Acosta, V. (2012). Algunos retos y propuestas en la conceptualización, evaluación e intervención del Trastorno Específico del Lenguaje (TEL). Revista Chilena de Fonoaudiología, 11, 23-36. https://doi.org/10.5354/07194692.2012.24525

American Speech Language Hearing Association. (2005). Audiology Information Series. ASHA.

Balbi, A., Cuadro, A., \& Trías, D. (2009). Comprensión lectora y reconocimiento de palabras. Ciencias Psicológicas, 3(2), 153-160.

Bishop, D., \& Adams, C. (1990). A prospective study of the relationship between specific language impairment, phonological disorders and reading retardation. Journal of Child Psychology and Psychiatry, and Allied Disciplines, 31(7), 1027-1050.

Catts, H., Fey, M., Tomblin, J. B., \& Zhang, X. (2002). A longitudinal investigation of reading outcomes in children with language impairments. Journal of Speech, Language, and Hearing Research: JSLHR, 45(6), 1142-1157.

Catts, H., \& Kamhi, A. (2005). Language and Reading Disabilities (3 edition). Boston: Pearson.

Coloma, C. J., \& De Barbieri, Z. (2007). Trastorno fonológico y conciencia fonológica en preescolares con trastorno específico del lenguaje. Revista de Logopedia, Foniatría y Audiología, 27(2), 67-73. https://doi.org/10.1016/S02144603(07)70075-X

Coloma, C. J., Pavez, M. M., Peñaloza, C., Araya, C., Maggiolo, M., \& Palma, S. (2012). Desempeño lector y narrativo en escolares con trastorno específico del lenguaje. Onomázein, 26(2), 351-375.

Coloma, C. J., Silva, M., Palma, S., \& Holtheuer, C. (2015). Comprensión lectora en niños con Trastorno Específico del Lenguaje: un estudio exploratorio de habilidades lingüísticas y de decodificación. Psykhe, 24(2), 1-8. https://doi.org/10.7764/psykhe.24.2.763 
Coloma, C. J., Sotomayor, C., De Barbieri, Z., \& Silva, M. (2015). Comprensión lectora, habilidades lingüísticas y decodificación en escolares con trastorno específico del lenguaje. Revista de Investigación en Logopedia, 5(1), 1-17.

Conti-Ramsden, G., Botting, N., Simkin, Z., \& Knox, E. (2001). Follow-up of children attending infant language units: outcomes at 11 years of age. International Journal of Language \& Communication Disorders, 36(2), 207-219.

Dickinson, D., McCabe, A., Anastasopoulos, L., PeisnerFeinberg, E. S., \& Poe, M. D. (2003). The comprehensive language approach to early literacy: The interrelationships among vocabulary, phonological sensitivity, and print knowledge among preschool-aged children. Journal of Educational Psychology, 95(3), 465-481. https://doi.org/10.1037/0022-0663.95.3.465

Fundación Chile, \& Centro de Estudios MINEDUC. (2013). Análisis de la Implementación de los Programas de Integración Escolar (PIE) en establecimientos que han incorporado Estudiantes con Necesidades Educativas Especiales Transitorias (Documento) (p. 40). Chile: Ministerio de Educación. Recuperado de https://especial.mineduc.cl/wp-

content/uploads/sites/31/2016/08/Resumen_Estudio_Imp lementacionPIE_2013.pdf

Guasti, M. T. (2002). Language Acquisition. Massachusetts: Cambridge, Mass.

Himmel, E., \& Infante, M. (2007). Construcción y validación del perfil de aprendizajes logrados en los sectores de lenguaje y matemáticas en los niveles NB1 y NB2 (Proyecto No. 1040985). Facultad de Educación, Pontificia Universidad Católica de Chile.

Hoover, W. A., \& Gough, P. B. (1990). The simple view of reading. Reading and Writing, 2(2), 127-160. https://doi.org/10.1007/BF00401799

Kelso, K., Fletcher, J., \& Lee, P. (2007). Reading comprehension in children with specific language impairment: an examination of two subgroups. International Journal of Language \& Communication Disorders, 42(1), 39-57. https://doi.org/10.1080/13682820600693013

Marinis, T. (2011). On the nature and cause of Specific Language Impairment: A view from sentence processing and infant research. Lingua, 121(3), 463-475. https://doi.org/10.1016/j.lingua.2010.10.010

Mesa, G., Tirado, M. J., \& Saldaña, D. (2013). El retraso en el desarrollo del lenguaje y los problemas de comprensión lectora: una exploración del modelo simple de lectura. Revista de Logopedia, Foniatría y Audiología, 33(3), 136145. https://doi.org/10.1016/j.rlfa.2012.06.001
Ministerio de Educación. Decreto Supremo 170 (2010). Recuperado de https://www.leychile.cl/Navegar?idNorma $=1012570$ Nation, K., \& Snowling, M. J. (2004). Beyond phonological skills: broader language skills contribute to the development of reading. Journal of Research in Reading, 27(4), 342-356. https://doi.org/10.1111/j.14679817.2004.00238.x

Oakhill, J. V., \& Cain, K. (2012). The Precursors of Reading Ability in Young Readers: Evidence From a Four-Year Longitudinal Study. Scientific Studies of Reading, 16(2), 91121. https://doi.org/10.1080/10888438.2010.529219

Pavez, M. M., Coloma, C. J., \& Maggiolo, M. (2008). El desarrollo narrativo en niños: una propuesta práctica para la evaluación y la intervención en niños con trastorno del lenguaje. Barcelona: Ars Medica.

Protopapas, A., Simos, P. G., Sideridis, G. D., \& Mouzaki, A. (2012). The Components of the Simple View of Reading: A Confirmatory Factor Analysis. Reading Psychology, 33(3), 217-240. https://doi.org/10.1080/02702711.2010.507626

R Core Team. (2018). R: A language and environment for statistical computing (Versión 3.4.4). Vienna, Austria. Recuperado de http://www.R-project.org/

Raven, J. C. (2005). Test de matrices progresivas. Escala coloreada, general y avanzada. Buenos Aires: Paidós.

Ricketts, J. (2011). Research review: reading comprehension in developmental disorders of language and communication. Journal of Child Psychology and Psychiatry, and Allied Disciplines, 52(11), 1111-1123. https://doi.org/10.1111/j.1469-7610.2011.02438.x

Sanz-Torrent, M., Andreu, L., Badia, I., \& Serra, M. (2010). El proceso lector en niños con antecedentes de retraso y trastorno específico del lenguaje. Revista de Logopedia,

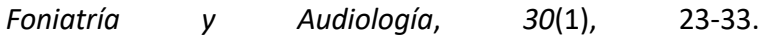
https://doi.org/10.1016/S0214-4603(10)70005-X

Snowling, M., Bishop, D. V., \& Stothard, S. E. (2000). Is preschool language impairment a risk factor for dyslexia in adolescence? Journal of Child Psychology and Psychiatry, and Allied Disciplines, 41(5), 587-600.

Vandewalle, E., Boets, B., Boons, T., Ghesquière, P., \& Zink, I. (2012). Oral language and narrative skills in children with specific language impairment with and without literacy delay: a three-year longitudinal study. Research in Developmental Disabilities, 33(6), 1857-1870. https://doi.org/10.1016/j.ridd.2012.05.004

Woodcock, R., Muñoz, A., Ruef, M., \& Alvarado, C. (2005). Language survey-revised. Test Book- Spanish. Rolling Meadows: Riverside Publishing Company. 